\title{
Handheld Imaging System for Contactless Tri-Modal Biometric Identification
}

\author{
S. Mil'shtein*, V. Oliyil Kunnil, C. McPherson , A. Pillai \\ Advanced Electronic Technology Center, ECE Dept., UMass, Lowell, MA-01854, USA
}

\begin{abstract}
Fingerprinting is the most widely used form of biometric identification because large databases are already available and law enforcement agencies have decades of experience with this technology. In addition, many fingerprinting units on the market are small in size, low power, and inexpensive. Common deficiencies carried by current fingerprinting technologies are distortions introduced when the finger touches the surface of the reader. The current study describes the development of a novel, handheld, contactless fingerprinting system which is capable of acquiring individual fingers with 180 degree view (first modality). Liveliness is examined by transmitted Infra-Red (IR) light (second modality), which allows the viewing of blood vessel networks and "four-slap" images and palm prints (third modality). This system is able to produce fingerprint images which are approximately $99 \%$ accurate representations of a finger's surface. This was verified by examining a NIST certified aluminum test sample. As demonstrated in our study, this is compared to an average of $15 \%$ distortion of the distances between a finger's ridges when imaged with contact based systems. Our radial line-scan imaging allows for "nail-to-nail" acquisition of an individual fingerprint, where the format of the recorded image is analogous to an "ink-rolled" fingerprint. The system will securely and wirelessly transfers fingerprint images to any law enforcement center via built-in Wi-Fi connection.
\end{abstract}

Keywords Multi-Modal Biometrics, Contactless Fingerprinting, Blood Vessel Mapping

\section{Introduction}

The major development of fingerprint technology in the form of wet-ink fingerprinting was initiated and improved for forensic applications by Scotland Yard about 100 years ago. However, the development of new fingerprinting methods has happened in recent years and continues to evolve. Fingerprint recognition technology is an integral part of criminal investigations. It is the basis for the design of numerous security systems in both private and public sector. It is also seen as an important tool for a variety of government organizations including Homeland Security, Immigration, Naturalization Services, and the Armed Forces, where fingerprinting procedures are used for recognition and verification of the identity for employees of federal departments and private contractors. In addition, the growth of the internet has made it necessary to verify the identity of individuals online.

After the tragic events of September 11, 2001, the need for improved and reliable fingerprint recognition technology drastically increased. We witnessed the replacement of wet ink fingerprinting by digitized contact-based methods.

* Corresponding author:

sam_milshtein@uml.edu (S. Mil'shtein)

Published online at http://journal.sapub.org/ajbe

Copyright (C) 2011 Scientific \& Academic Publishing. All Rights Reserved
Biometrics is a rapidly growing industry which is anticipated to grow upwards of $20 \%$ per year through 2015 [1]. Fingerprinting is the most widely used form of biometric identification because large databases are already available and law enforcement agencies have decades of experience with this technology. In addition, many fingerprinting units on the market are small in size, low power, and inexpensive. Common deficiencies carried by current fingerprinting technologies are distortions introduced when the finger touches the surface of the reader. To the contrary, unfitness of contactless fingerprints capturing is the ability to store the fingerprint which is almost $100 \%$ distortion free. Various applications for law enforcement purposes define the portion of a finger, number of fingers to be captured, section of a palm and most important the format of biometric images. It is current FBI requirements of 700 ppi and coming soon 1000 ppi formats, as well as NIST standards[2], regulate the design of the hardware and actual fingerprinting procedures. The current study describes the development of a novel, handheld, contactless fingerprinting system which is capable of acquiring "four-slap" images, palm prints, and individual fingers with 180 degree view.

In addition, liveliness of all three objects is examined by transmitted Infra-Red (IR) light, which allows the viewing of blood vessel networks. This system is able to produce fingerprint images which are approximately 99\% accurate representations of a finger's surface. We demonstrated in[3] 
that touching the fingerprint scanner introduce an average of $15 \%$ distortion of the distances between a finger's ridges.

Ability of our system was verified by examining a NIST certified aluminum test sample. Our system utilizes the advantages of radial line-scan imaging developed recently[4] for contactless fingerprinting and blood vessel imaging. Our technique allows for "nail-to-nail" acquisition of an individual fingerprint, where the format of the recorded image is analogous to an "ink-rolled" fingerprint. The system will securely and wirelessly transfers fingerprint images to any law enforcement center via built-in Wi-Fi connection. The study describes a fully automatic system and assesses the technical and marketability aspects of this novel product. The systems secure interface to law enforcement databases will also be discussed. Although, contactless methods generate highest quality of fingerprints, the utilization of this technology requires additional study which will compare the contactless methods with current digitized and wet-ink libraries.

\section{Design of System}

All the proposed methods of fingerprint capturing can be grouped into two major families: solid-state readers and optical readers, i.e. CCD arrays and computer scanners. Our patents [5-6] describes stationary contactless line scanning system with Infra-Red (IR) light performing second biometric check on the map of blood vessels. However the operation of the system is based on mechanical move of heavy optical system including set of lenses and line scanning camera. Our system uses single array of CCDs.

Our system combines a novel method of radial line-scan imaging with a separate and independent camera system for use as a handheld, four-modal, biometric image acquisition system. The first modality is a nail-to-nail fingerprint captured using the radial line scan hardware. The second modality is a blood vessel image which is recorded via transmitted Infra-Red (IR) light using the same radial line scan imaging hardware used for fingerprint capture. The third modality is a 4-slap print and a palm print which is captured using an area scan imaging system. The developed radial line scan technique allows for this device to fit in a volume which is typical of handheld devices, $15 \mathrm{~cm} \times 13 \mathrm{~cm} \times 17 \mathrm{~cm}(6.3 \mathrm{in}$. $\mathrm{x} 5.1$ in $\times 6.7 \mathrm{in}$ )

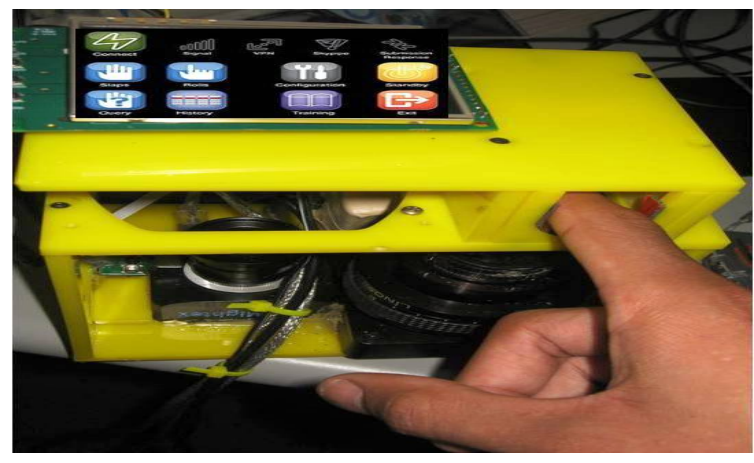

Figure 1. Handheld fingerprinting system
The novel hand-held contactless system has all optical components and its line-scan camera rigidly mounted to the machines chassis, i.e. no moving parts. In this configuration all optical components and optical paths remain fixed in position relative to the scanner body. This is accomplished using a line-scan camera and large aperture lens system that directly views the fingers surface from a distance of $5 \mathrm{~cm}$. Each captured fingerprint image is created by first capturing $1 \times 2048$ pixel images (lines) as the finger rotates directly above the lens system. Over the course of the finger rotating 180 degrees over 1000 lines are collected. These images are then stitched together to form the final fingerprint image.

The optics and other components within the scanner are rigidly mounted within a rigid plastic structure. The scanner chassis has been optimized to reduce the overall weight of the machine, as well as the size of its construction.

For accurate image construction, it is important to tag each specific line with the location on the finger that they are an image of. For this purpose, the finger is positioned in an alignment system that is able to record the angular position of the finger over the course of a scan. This is accomplished using an optical encoding system. The angular position of the finger over time can be fully resolved from the data originating from this system.

\section{Fingerprinting and Blood Vessels}

The fingerprinting procedure is completely automated. On request of the officer performing fingerprinting, the individual to be fingerprinted will put one by one each finger for $180 \mathrm{o}$ line-scan. This gray-scale fingerprint is binarized as shown in Fig.2 and the fingerprint image is transmitted together with its binarized version. The system utilizing special command will submit wirelessly the image of the finger. Then, 4-slap image and palm prints, as illustrated by Fig. 3, taken by the area scan camera, would be transmitted in a similar way to the central database. Finally, the blood vessels of 4-slap fingers, which can be seen in Fig. 4, will be submitted, thus verifying the liveliness of the fingers. This verification proves that no latex decoy of the fingers was used. For mapping of blood vessels, the LED chip carrying 66 light emitters generates the infrared light with wavelength $\lambda=910 \mathrm{~nm}$. The image of blood vessel is registered in transmitted light.

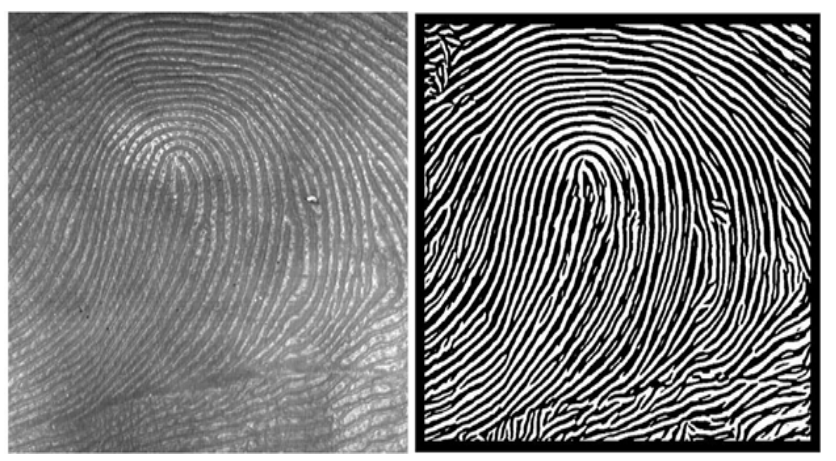

Figure 2. Grayscale image and its binarized image 


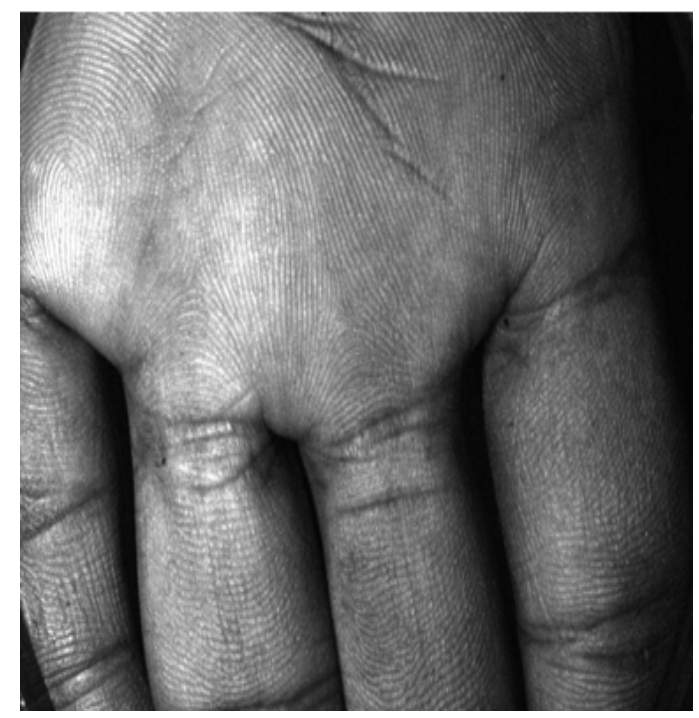

Figure 3. 4-slap image.

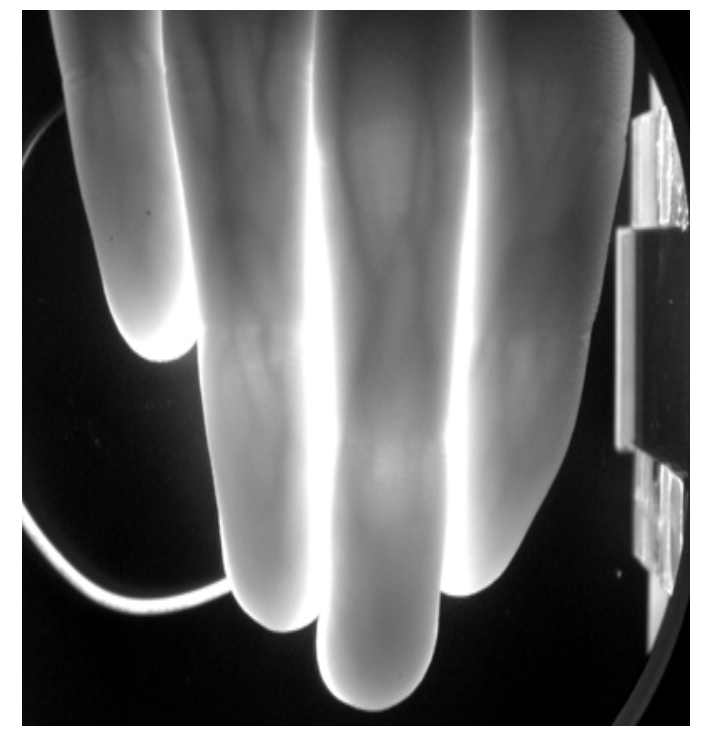

Figure 4. Blood vessel map

\subsection{Binarization Procedure}

Most fingerprint recognition algorithms rely on the clarity and details of ridges. Hence, it is necessary to clearly differentiate the fingerprint ridges and valleys using only two distinct values; this process is called binarization. Regardless of the quality of any image recognition algorithm, a poorly binarized image can compromise its recognition statistics.

A good binarization algorithm would produce an image which would have very clear and uniform black ridges on a white background even if the image is overexposed to a certain degree. We used the following binarization techniques:

1. Region-Based thresholding as described below.

2. Filter-Based technique.

The region based thresholding starts with division of the image into an N-by-N grid of smaller blocks. Identification of ridge regions within these smaller blocks is performed. This is implemented by taking the gradients in the $\mathrm{x}$ and $\mathrm{y}$ direction and then finding the covariance data for the image gradients. Once this step is completed, the orientation of ridges is computed by finding the angle with respect to the coordinate axis. Then, estimation of ridge frequencies in these blocks is performed. This is done to find out which blocks have a higher and a lower density of ridges. The image block is then rotated to make the ridges vertical, and is cropped to remove invalid regions. A projection of the grayscale values, down the ridges, is obtained by summing along the columns.

Peaks in projected grey values are found by performing dilation and finding where the dilation equals the original values. The spatial frequency of the ridges is determined by dividing the distance between the 1st and last peaks by the number of peaks. If no peaks are detected, or the frequency of ridge occurrence is outside the allowed bounds, the frequency is set to 0 . The information about ridge regions, orientation and frequencies returns a mask of a fixed size which defines the actual area where the fingerprint exists. The ridges are then enhanced with the help of a median filter. The image obtained after this process is thresholded to obtain the binary fingerprint. The threshold for binarization depends on the resolution for the image. This process can also be called Adaptive Binarization. This method works very well with the images that are obtained from the contactless fingerprinting system described in this manuscript. This binarization technique is not affected by varying brightness levels throughout the image, and results in a binary image that has consistent information throughout.

\section{Applications of Contactless Fingerprinting Technology}

Increased security threats with respect to terrorism and cyber-crime have recently necessitated the development of biometric systems to be used at commercial facilities, border crossings, airports, and government building access points. Additionally, fraud with credit card accounts, hacking of retail store websites, and most importantly, the critical interruption of governmental agencies such as the Department of Defense and the Department of Homeland Security, requires the development of systems capable of identifying individuals accurately to mitigate such attacks. When automated fingerprinting systems were introduced in the late 1960s, digital contact-based fingerprinting replaced the old method of ink rolling. This facilitated a new range of fingerprinting applications. The increased accuracy of contactless fingerprinting will create new applications in fingerprinting as well. Specifically, applications will be found in the fields of information security, access control, and law enforcement. Below are some examples of how contactless fingerprint systems can be used in both the private and the public sector.

\subsection{Law Enforcement Agencies}

Every organization has unique requirements for stored 
fingerprints, depending on how these prints are utilized. For example, agencies that deal with crime scene fingerprints prefer to have nail-to-nail images, because crime scene images may be partial; and the more information available at registration stage helps in matching them with partial prints lifted from a crime scene. This specific need of individual organizations has resulted in different agencies having their own unique and often incompatible databases. Recently, creation of a unified and accurate database across all agencies has been recognizedas a necessary step in the evolution of law enforcement's capabilities. A standardized method which captures a nail-to-nail image will help these agencies migrate towards a single large database, from which a specific portion of the image may be extracted depending on individual requirements. To make this step, a new standard of fingerprints and fingerprinting hardware will need to be developed and followed across all agencies. Implementation details of such project will not be discussed here; however applications of such a database will be explored. Currently, it is impossible for law enforcement to identify an individual in real-time based on fingerprints only. This is partially due to a lack of computer processing power to sort through databases containing millions of images. Most important, the current databases contain fingerprints taken using contact-based methods, and thus have varying degrees of distortion. A database system containing high quality images and a repeatable method for fingerprint capture would facilitate applications such as real time recognition of individuals. For example, police officers carrying mobile fingerprint capture units can successfully execute an arrest as soon as he verifies the identity of the individual.

\subsection{Access Control}

Access control can also benefit from such devices. Current fingerprint based access control devices have a certain disadvantage in usability. Often a user may need to repeatedly scan their finger before they are granted access. The need to use a finger few times is caused by inconsistencies between the fingerprint data recorded by the capture device and the data stored within the system's database. This inconsistency increases the systems margin of error, translates to increased false rejections and a lower degree of confidence with every match. In high security access control, an additional measure can be taken to further increase the degree of confidence with every match. Spoof detection is a technique that focuses on determining whether a finger is currently alive and attached to the body, and is in fact the person's real finger.

\subsection{Financial Transaction}

In the commercial sector, accurate biometric based authentication can be implemented in electronic commerce and confidential email exchange. Methods of authentication such as tokens, cards, badges, passwords and pins are widely being used today. These methods can be supplemented by accurate fingerprint based authentication to obtain a higher degree of user confidence, as well as decrease the presence of fraud in online spaces. At places of financial transactions, Automatic Teller Machines, and E-commerce are all areas that can potentially find solutions to long-standing security related problems through the use of commercialized contactless fingerprinting devices.

\section{Conclusions}

For the first time, a multi-modal contactless fingerprinting system was designed and built. This system is capable of taking high resolution images of ten fingers with $180 \mathrm{o}$, nail-to-nail scan of every finger separately. Sequentially four slap and palm of the same hand can be taken in a contactless manner. Finally, mapping of blood vessels is performed for verifying liveliness of the fingers. Contactless fingerprinting produces the best quality, non-distorted fingerprint images.

This technology[7-8] has variety of application like perimeter access, airports and border crossing and securing network transactions. The list of potential applications where non-distorted and repeatable fingerprints could be used is much longer. Small dimensions of the equipment, autonomous wireless communication to data centers make this handheld system very useful in police cruisers, border patrols and other mobile law enforcement units.

\section{REFERENCES}

[1] Global Biometrics Technology Market 2010-1015. Market Forecast by Products, End-User Application and Geography, Report, marketsandmarkets.com, SE 1302 , Jan (2011)

[2] McCabe R. and Garris M., Summary of April 2005 ANSI/NIST Fingerprint Standard Update Workshop, 2005

[3] S. Mil'shtein, U. Doshi, Scanning of the Pressure-Induced Distortion of Fingerprints, Scanning, 26, 4, pp: 323-327, 2004

[4] S. Mil'shtein, J. Palma, C. Liessner, M. Baier, A. Pillai, and A.Shendye, Line Scanner for Biometric Applications, IEEE Intern. Conf. on Technologies for Homeland Security, ISBN 978-1-4244-1978-4 P 205-208, 2008.

[5] S. Mil'shtein, J. Palma, C. Liessner, Circumferential Contact-less Line Scanning of Biometric Objects, Patent \# 60,840,550, September, 2006

[6] S. Mil'shten, M. Baier, A. Pillai, A. Shendye, Simultaneous Identification of Fingerprints and Mapping of Blood Vessels in a Finger, Patent application, 2009

[7] M. Baier, S. Foret, V. Oliyil Kunnil, M. Paradis, P. Bustos, and S. Mil'shtein, Automatic Contactless Mobile Fingerprinting System, Proc. of VISAPP 2011, Portugal, Mar. 2011

[8] S. Mil'shtein, M. Paradise, P. Bustos, M. Baier, S. Foret, V. O. Kunnil, J. Northrup, Contactless Challenges, Biometr. Techno. Today, pp: 10-11, Mar. 2011 